\title{
matters \\ Ammonium carbonate gas diffusion crystallization in a continuous organic medium leads to dendritic calcium carbonate
}

\author{
$\checkmark$ Correspondence \\ eddie.peh@mpikg.mpg.de \\ 8 Disciplines \\ Mineralization \\ Q Keywords \\ Calcium Carbonate \\ Dendrites \\ Ammonium Chloride \\ Crystallization \\ Optical Microscopy \\ it Type of Observation \\ Standalone \\ \& Type of Link \\ Standard Data \\ (O) Submitted Apr 27, 2017 \\ (1) Published Sep 14, 2017
}

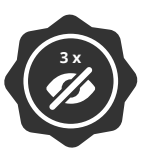

Triple Blind Peer Review The handling editor, the reviewers, and the authors are all blinded during the review process.

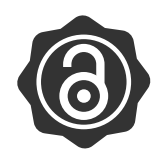

Full Open Access

Supported by the Velux Foundation, the University of Zurich, and the EPFL School of Life Sciences.

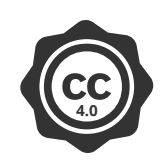

Creative Commons 4.0 This observation is distributed under the terms of the Creative Commons Attribution 4.0 International License.

\section{Eddie Peh, Andreas Taubert, Klaus Tauer}

Colloid chemistry, Max Planck Institute of Colloids and Interfaces; Institute of Chemistry, University of Potsdam; Colloid Chemistry, Max Planck Institute of Colloids and Interfaces

\begin{abstract}
The crystallization of calcium carbonate in a continuous organic medium using the classic ammonium carbonate gas diffusion system was recorded with an optical microscope. Surprisingly, dendritic structures were obtained consistently when the product gas mixture combined with calcium chloride already dissolved in the organic medium. Characterization studies revealed these crystals to be composite mixtures of calcium carbonate and ammonium chloride.
\end{abstract}

\section{Objective}

To study calcium carbonate crystallization in a continuous organic medium containing dissolved calcium chloride while using ammonium carbonate gas diffusion method to feed the reaction precursors.

\section{Introduction}

To the best of our knowledge, till date, crystallization studies of calcium carbonate have always been carried out either in pure water or in homogeneous mixtures of water with hydrophobic organic solvents [1] [2]. The main role of water is to dissolve the individual precursor salts in order to form the solvated calcium cations and carbonate anions respectively. There are currently no reports on using pure organic solvents for calcium carbonate formation. For this purpose, considering Coulomb's equation, organic solvents withvery high dielectric constants are preferred. Among the potential compounds, propylene carbonate $(\mathrm{PC})$ is one of the suitable candidate with a dielectric constant of about 65 [3]. The ammonium carbonate gas diffusion method provides an advantage when calcium chloride is dissolved in the organic solvent, because the evaporation products $\left(\mathrm{CO}_{2}, \mathrm{NH}_{3}\right.$, and $\left.\mathrm{H}_{2} \mathrm{O}\right)$ have a rather high solubility in organic solvents [4]. For every mole of ammonium carbonate salt that decomposes, two moles of ammonia, one mole of carbon dioxide and in fact one mole of water are produced. The water formation is unimportant in aqueous systems but essential during calcium carbonate crystallization in pure organic continuous phases for the formation of the corresponding precursor ions $\left(\mathrm{NH}_{4}{ }^{+}, \mathrm{CO}_{3}{ }^{2-}\right)$.

We have used a setup as shown in figure $\mathrm{S}_{1}$ (in supplementary information) allowing on-line monitoring of the crystallization process with optical microscopy. For this, an optical glass cuvette filled with a calcium chloride in PC solution is connected via a tubing to a glass vial containing $0.5 \mathrm{~g}$ of ammonium carbonate. We were able to dissolve only sparingly soluble calcium chloride up to $0.2 \mathrm{wt}$.\% in PC. Solid ammonium carbonate gradually decomposes into gaseous carbon dioxide, ammonia, and water. The decomposition products diffuse into the cuvette, driven by chemical potential differences. In order to facilitate the diffusion, the vial with the ammonium carbonate is put above the cuvette considering that carbon dioxide is denser than ambient air. Using a special holder, the cuvette is placed upright in front of the objective of the optical microscope that is subsequently connected to a computer allowing real time imaging of the cuvette. All experiments have been carried out at room temperature. 


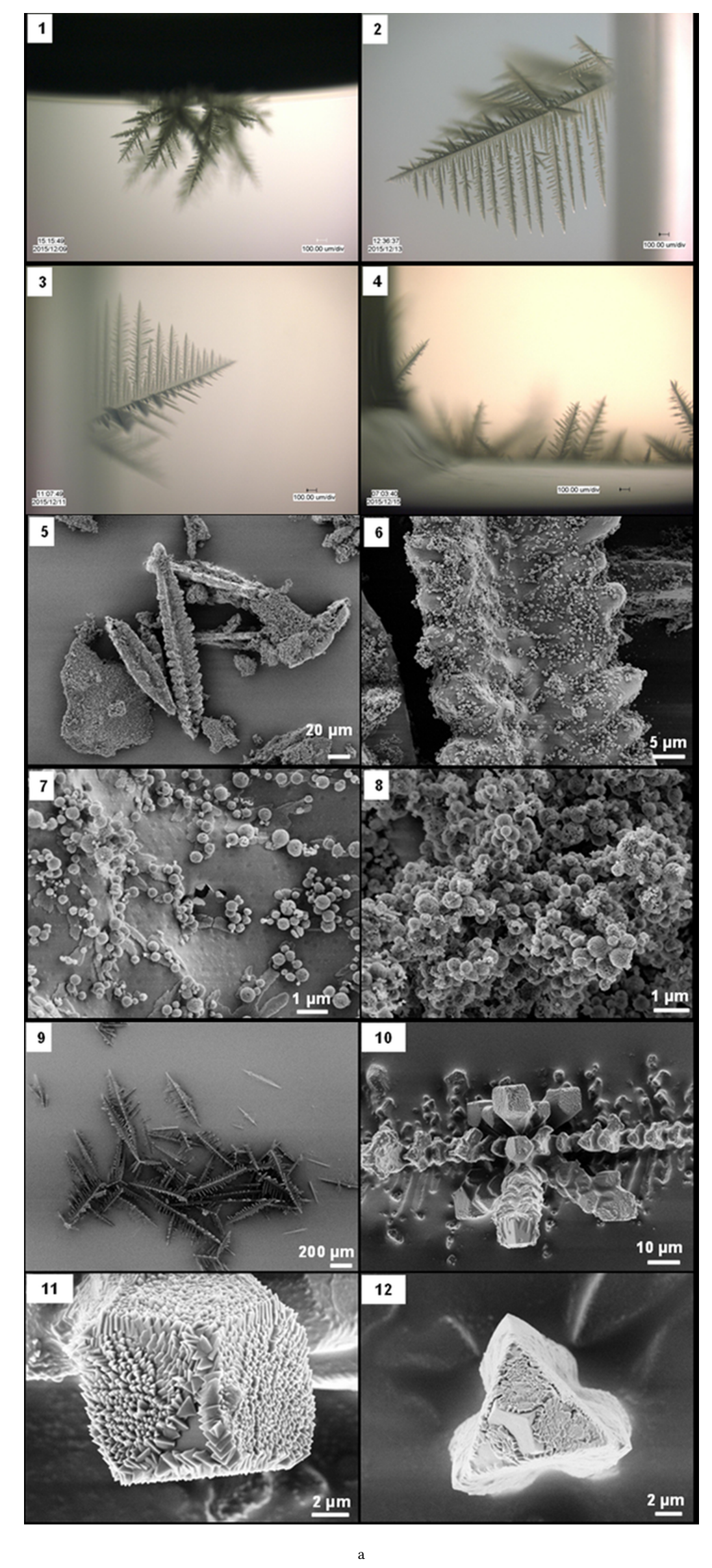

CARBONATE 


\section{Figure Legend}

Figure A:

Optical microscope (1-4): Images of dendritic crystals grown on (1) air-organic interface $(2,3)$ middle section and $(4)$ bottom section of the glass cuvette, internal diameter $=10 \mathrm{~mm}$.

Scanning Electron Microscopy (5-12): Images of isolated dendritic crystals grown in PTFE vessel filled with propylene carbonate saturated with $\mathrm{CaCl}_{2}$ (0.2 wt.\%).

As additional information, high-resolution images are provided separately as raw data file.

\section{Results \& Discussion}

Typical crystals of calcium carbonate exhibit rhombohedral, orthorhombic or hexagonal crystal structures depending on the polymorph generated under the particular experimental conditions with respect to temperature, composition, and concentration [5]. Surprisingly, under the experimental conditions in this study, only dendritic crystals have been detected by optical microscopy, cf. images put together in figure $\mathrm{A}_{1-4}$ (taken after 30 hours of reaction time). The multiple dendritic crystals had grown exclusively from interfaces, either from the vapor-PC interface (figure $\mathrm{A}_{1}$ ) or from all sites of the glass-PC interface (figures $\mathrm{A}_{2}-4$ ), and stretched into the PC phase. In all repeats, dendrites were the only crystals detected with optical microscopy. In general, these crystals resemble to the shape of pine trees. However, it also may happen that some lower primary branches are shorter than others grown nearer the tip, probably due to space constraints imposed by neighboring main trunks. In addition, secondary branches were also growing from the primary branches and their maximum size appeared to be also limited by the interspacing between primary branches.

In order to check on the influences of higher supersaturation levels, experiments were also performed in glass cuvettes but with smaller internal diameters. In general, the overall number of crystals obtained in cuvettes with 1 and $5 \mathrm{~mm}$ thickness was much higher but their sizes were smaller, only in the range of 50 to $100 \mu \mathrm{m}$ compared to the 10 $\mathrm{mm}$ cuvette. We also found that the general dendritic structure was preserved, but the pine tree shape has changed. In most cases the crystals exhibited patterns with trigonal, tetrahedral and occasionally octahedral symmetries corresponding to the number of main trunks that had grown from a single nucleation site (cf. figure S2).

To investigate the structure of the dendritic crystals, X-ray powder diffraction analysis was performed. Figure $S_{3}$ shows a typical XRD pattern of the crystals. The XRD pattern clearly reveals a composite structure of the crystals, a distinct difference to corresponding experiments carried out in aqueous continuous phases. However, this is not a big surprise, since in aqueous systems the solubility product of ammonium chloride is several orders of magnitude higher than that of calcium carbonate, but is obviously not the case for PC as the continuous phase. The solubility of ammonium chloride in water is $39.5 \mathrm{~g} / 100 \mathrm{~g} \mathrm{H}_{2} \mathrm{O}$ while that of calcium carbonate is $0.001 \mathrm{~g} / 100 \mathrm{~g} \mathrm{H}_{2} \mathrm{O}$ [6] [7]. In contrast, it might be considered as surprise that the calcium carbonate polymorph is vaterite and not the thermodynamically more stable calcite. The presence of vaterite and ammonium chloride was reaffirmed with Fourier transform infrared spectroscopy, Raman spectroscopy and elemental analysis (cf. figure $S_{4}-6$ ). These results confirm the above conclusion that the dendrites are essentially composite structures made of ammonium chloride and vaterite.

Considering that ammonium chloride is a kind of standard textbook example for dendrite formation [8] [9], the generation of composite dendrites in the experiments described here is not surprising. Dendrites are structures formed in the presence of diffusion limitations that are present during crystal growth [10] [11]. These diffusion irregularities often lead to higher supersaturations at specific areas such as corners and edges ,and the resulting faster growth rates generate the final dendritic morphology [12]. Like ice crystals, ammonium chloride also possesses a crystalline anisotropy that favors the formation of dendrites during its crystallization [13].

To obtain more material and information about the growth mode, experiments were repeated in larger containers made of PTFE. Figures $\mathrm{A}^{-12}$ show scanning electron micrographs of several isolated dendrites and reveal their composite structure. Figure $\mathrm{A}_{5}$ 
shows the main trunk of one dendrite reaching to about $100 \mu \mathrm{m}$ in height. The definitions of the primary branches and the interspacing between each branch have significantly diminished indicating a high level of overgrowth. In addition, many clusters of spherical structures with diameters up to $1 \mu \mathrm{m}$ can be seen clearly deposited on the exterior surface of the dendrites (cf. figure A6-8). These clusters resemble very closely to the vaterite particles synthesized in several works [14] [15] [16].

Judging from the significant difference between crystal sizes, $100 \mu \mathrm{m}$ dendrites and only $1 \mu \mathrm{m}$ spherical clusters, the growth mechanism may then be formulated as a two stage process. During the decomposition of ammonium carbonate, both ammonia and carbon dioxide gases combine with water to form the respective ammonium $\left(\mathrm{NH}_{4}^{+}\right)$and carbonate $\left(\mathrm{CO}_{3}{ }^{2-}\right)$ ions. Assuming similar diffusion speed and solubility, the propylene carbonate phase becomes saturated first with respect to ammonium chloride which thus nucleates and grows as the first stage. This suggestion that ammonium chloride precipitate first is supported by the stoichiometry of the ammonium carbonate decomposition which leads to two moles of ammonia for every mole of carbon dioxide produced. In addition, the affinity of ammonia to water molecules is much higher due to hydrogen bonding compared to its counterpart [17]. As the dendritic ammonium chloride crystals are growing, the propylene carbonate phase is also reaching the supersaturation with respect to calcium carbonate, in this case the less stable polymorph vaterite. The precipitated ammonium chloride dendrites act as seed crystals and provide interfaces that favor the subsequent nucleation and thereby growth of vaterite crystals.

The SEM micrographs of figure A9-12 illustrate the composite nature of the dendrites quite clearly with a shell made of many triangular calcium carbonate crystallites of sizes up to $600 \mathrm{~nm}$ overlaying in a scaly fashion an inner homogenous almost monolithical ammonium chloride core (cf. figure A11). This microscopic structure is in accordance with the macroscopic observation that the dendrites collapse after water addition (cf. figure $\mathrm{S}_{7}$ ).

\section{Conclusions}

In conclusion, this is the first time calcium carbonate is crystallized in a continuous organic medium. Unexpectedly, well defined dendritic crystals were obtained consistently. Characterization studies revealed the crystals to be composite-like made of vaterite and ammonium chloride.

\section{Limitations}

Characterization of single dendrites grown is not trivial due to their poor mechanical and water stability.

\section{Additional Information}

Methods and Supplementary Material

Please see https://sciencematters.io/articles/20170600oo12.

Funding Statement

We would like to acknowledge financial support from the Max Planck Institute of Colloids and Interfaces. In addition, E.P. acknowledges a scholarship from the IMPRS on Multiscale Bio-systems.

Ethics Statement

Not Applicable.

\section{Citations}


[1] Sommerdijk et al. "Biomimetic $\mathrm{CaCO}_{3}$ Mineralization using Designer Molecules and Interfaces". In: Chemical Reviews 108.11 (2008), pp. 4499-4550. URL: http:

//dx.doi.org/10.1021/cr078259o\%20http: //pubs.acs.org/doi/pdfplus/10.1021/ cr078259o.

[2] Meldrum and F. C. "Calcium carbonate in biomineralisation and biomimetic chemistry". In: International Materials Reviews 48.3 (2003), pp. 187-224. URL: http:

//dx.doi.org/10.1179/095066003225005836.

[3] Hong et al. "Some thermodynamic properties of systems containing propylene carbonate and ethylene carbonate". In: fournal of Chemical and Engineering Data 27.2 (1982), pp. 146-148. URL: http :

//dx.doi.org/10.1021/je00028a012\%20http: //pubs.acs.org/doi/pdf/10.1021/ je00028a012.

[4] Fogg and Peter. "Carbon Dioxide in Non-Aqueous Solvents At Pressures Less Than 200 KPA”. In: Solubility Data Series A2 50 (1992), p. ii. URL: / / wWW. sciencedirect. com/ science/article/pii/B9780080404950500014.

[5] Declet, A; Reyes, and E; Suarez O.M. "Calcium Carbonate Precipitation: A Review Of The Carbonate Crystallization Process And Applications In Bioinspired Composites". In: Reviews on Advanced Materials Science 44.1 (2016), pp. 87-107.

[6] Haynes and William M. "CRC Handbook Of Chemistry And Physics”. In: CRC press 95th Edition (2014).

[7] L. Niel Plummer and Eurybiades Busenberg. "The solubilities of calcite, aragonite and vaterite in $\mathrm{CO}_{2}-\mathrm{H}_{2} \mathrm{O}$ solutions between $\mathrm{o}$ and $90^{\circ} \mathrm{C}$, and an evaluation of the aqueous model for the system $\mathrm{CaCO}_{3}-\mathrm{CO}_{2}-\mathrm{H}_{2} \mathrm{O}$ ". In: Geochimica et Cosmochimica Acta 46.6 (1982), pp. 1011-1040. URL:

http://www.sciencedirect.com/science/ article/pii/0016703782900564.

[8] Honjo et al. "New Experimental Findings in Two-Dimensional Dendritic Crystal-Growth". In: Physical Review Letters 55.8 (1985), pp. 841-844. URL: \%3CGo\%20 to\%20 IS I \%3E : //WOS : A1985ANX2600020\%20http:

//journals.aps.org/prl/abstract/10.1103/ PhysRevLett.55.841.

[9] Raz et al. "Dendritic growth of ammonium chloride crystals: Measurements of the concentration field and a proposed nucleation model for growth". In: Physical Review A 40.2 (1989), pp. 1088-1095. URL: https :

//www.ncbi.nlm.nih.gov/pubmed/9902234.
[10] Gorbich et al. "Dendritic growth of snow crystals". In: Crystallography Reports 50.3 (2005), pp. 499-503. URL: http: //download.springer.com/static/pdf/ 43/art\%3A10.1134\%2F1.1927616.pdf? originUrl=http: //link. springer.com/ article/10.1134/1.1927616\&token $2=\exp =$ $1450205522 \sim a c l=/$ static/pdf/43/art\%253A10 . 1134\%252F1.1927616.pdf?originUrl=http\%3A\% 2F\%2Flink.springer. com\%2Farticle\%2F10.

$1134 \% 2 \mathrm{~F} 1.1927616^{*} \sim \mathrm{hmac}=$ 5ed8b7052c9712610d879a8ea173235544851d313f31375f99e 0

[11] Ben-Jacob Eshel and Garik Peter. "The formation of patterns in non-equilibrium growth". In: Nature 343.6258 (1990), pp. 523-530. DOI: $10.1038 / 343523 \mathrm{a} 0$. URL:

https://doi.org/10.1038/343523a0.

[12] Etsuro Yokoyama and Toshio Kuroda. "Pattern formation in growth of snow crystals occurring in the surface kinetic process and the diffusion process". In: Physical Review A 41.4 (1990), pp. 2038-2049. URL: https: / / www . ncbi.nlm.nih. gov/pubmed/9903314\%20http://journals.aps . org/pra/abstract/10.1103/PhysRevA.41.2038.

[13] Haruo Honjo, Shonosuke Ohta, and Mitsugu Matsushita. "Irregular Fractal-Like Crystal Growth of Ammonium Chloride". In: Fournal of the Physical Society of Japan 55.8 (1986), pp. 2487-2490. URL: \%3CGo\%20to\%20 ISI\%3E : / /WOS : A1986E537900003.

[14] Rodriguez-Blanco et al. "The kinetics and mechanisms of amorphous calcium carbonate (ACC) crystallization to calcite, viavaterite". In: Nanoscale 3.1 (2011), pp. 265-271. URL: http //dx.doi.org/10.1039/C0NR00589D\%20http: //pubs.rsc.org/en/content/articlepdf/ $2011 / \mathrm{nr} / \mathrm{c} 0 \mathrm{nr} 00589 \mathrm{~d}$.

[15] Ralf Beck and Jens-Petter Andreassen. "Spherulitic Growth of Calcium Carbonate". In: Crystal Growth and Design 10.7 (2010), pp. 2934-2947. URL: http : //dx.doi.org/10.1021/cg901460g\%20http: //pubs.acs.org/doi/pdfplus/10.1021/ cg901460g.

[16] Radek Ševčík et al. "Characterization of vaterite synthesized at various temperatures and stirring velocities without use of additives". In: Powder Technology 284 (2015), pp. 265-271. URL: http://www. sciencedirect.com/science/ article/pii/s0032591015005239\%20http: //ac.els-cdn.com/S0032591015005239/1s2.0-S0032591015005239-

main.pdf?_tid=f14769b6-7a53-11e6-a090$00000 \mathrm{aab} 0 \mathrm{f} 26 \& \mathrm{acdnat}=1473841343$ a0f08e83ec6ad455710bc8d6c071a92b.

[17] John A. Dean. "Lange's Handbook of Chemistry". In: McGraw-Hill 13th Edition (1985). 